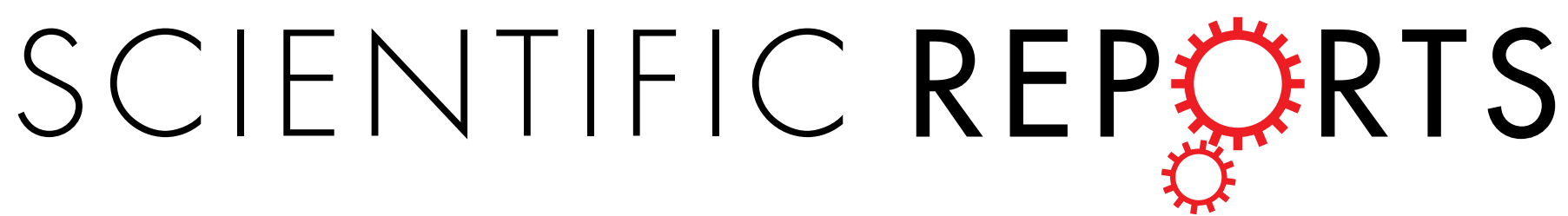

\title{
OPEN Does von Willebrand factor improve the predictive ability of current risk stratification scores in patients with \\ atrial fibrillation?
}

Received: 06 October 2016

Accepted: 15 December 2016

Published: 30 January 2017

\author{
Amaya García-Fernández ${ }^{1}$, Vanessa Roldán ${ }^{2}$, José Miguel Rivera-Caravaca ${ }^{3}$, \\ Diana Hernández-Romero ${ }^{3}$, Mariano Valdés ${ }^{3}$, Vicente Vicente ${ }^{2}$, GregoryY. H. Lip ${ }^{4,5, *}$ \& \\ Francisco Marín ${ }^{3, *}$
}

Von Willebrand factor (vWF) is a biomarker of endothelial dysfunction. We investigated its role on prognosis in anticoagulated atrial fibrillation (AF) patients and determined whether its addition to clinical risk stratification schemes improved event-risk prediction. Consecutive outpatients with non-valvular AF were recruited and rates of thrombotic/cardiovascular events, major bleeding and mortality were recorded. The effect of vWF on prognosis was calculated using a Cox regression model. Improvements in predictive accuracy over current scores were determined by calculating the integrated discrimination improvement (IDI), net reclassification improvement (NRI), comparison of receiveroperator characteristic (ROC) curves and Decision Curve Analysis (DCA). 1215 patients (49\% males, age 76 (71-81) years) were included. Follow-up was almost 7 years. Significant associations were found between vWF and cardiovascular events, stroke, mortality and bleeding. Based on IDI and NRI, addition of vWF to $\mathrm{CHA}_{2} \mathrm{DS}_{2}$-VASc statistically improved its predictive value, but c-indexes were not significantly different. For major bleeding, the addition of vWF to HAS-BLED improved the c-index but not IDI or NRI. DCA showed minimal net benefit. vWF acts as a simple prognostic biomarker in AF and, whilst its addition to current scores statistically improves prediction for some endpoints, absolute changes and impact on clinical decision-making are marginal.

Atrial fibrillation (AF) is increasingly more common and confers a five-fold increase in the risk of stroke ${ }^{1}$. AF patients also show high incidence of other cardiovascular events, such as acute coronary syndrome and cardiovascular death $^{2}$ and a residual risk remains despite the use of oral anticoagulation (OAC) ${ }^{3}$. When compared to control, OAC with Vitamin K antagonists (VKA) reduces the risk of stroke by $64 \%$ and all-cause mortality by $26 \%$, with further reductions using non-VKA OACs (NOACs) $)^{4,5}$.

The risks of thromboembolism and bleeding in $\mathrm{AF}$ are heterogeneous and several risk stratification schemes have been developed to tailor the decision-making. Guidelines ${ }^{6,7}$ recommend the use of the $\mathrm{CHA}_{2} \mathrm{DS}_{2}$-VASc score [Congestive heart failure, Hypertension, Age $\geq 75$ years, Diabetes mellitus, Stroke, Vascular disease, Age 65 to 64 years, Sex category], as a simple, clinical risk factor-based approach to thromboprophylaxis ${ }^{8}$. This score has been validated in different cohorts and we have previously demonstrated that it is also predictive for vascular events and mortality in $\mathrm{AF}^{9}$. Nevertheless, the predictive value of the $\mathrm{CHA}_{2} \mathrm{DS}_{2}$-VASc and other clinical factor-based risk stratification schemes for identifying 'high risk' patients that develop events remains modest ${ }^{10}$. Nonetheless, the $\mathrm{CHA}_{2} \mathrm{DS}_{2}$-VASc has shown to reliably identify AF patients at truly low-risk of thromboembolism, who require no

${ }^{1}$ Cardiology Service, Alicante University General Hospital, Alicante Institute for Health and Biomedical Research (ISABIAL - FISABIO Foundation), Alicante, Spain. ${ }^{2}$ Department of Hematology and Clinical Oncology, Morales Meseguer University Hospital, University of Murcia, Biohealth Research Institute Virgen de la Arrixaca, IMIBArrixaca, Murcia, Spain. ${ }^{3}$ Department of Cardiology, Virgen de la Arrixaca University Hospital, University of Murcia, Biohealth Research Institute Virgen de la Arrixaca, IMIB-Arrixaca, Murcia, Spain. ${ }^{4}$ University of Birmingham Institute of Cardiovascular Sciences, City Hospital, University of Birmingham, Birmingham, United Kingdom. ${ }^{5}$ Aalborg Thrombosis Research Unit, Department of Clinical Medicine, Aalborg University, Aalborg, Denmark. ${ }^{*}$ These authors jointly supervised this work. Correspondence and requests for materials should be addressed to V.R. (email: vroldans@gmail.com) 
antithrombotic therapy ${ }^{11}$. However, use of $\mathrm{CHA}_{2} \mathrm{DS}_{2}$-VASc results in a high proportion of patients treated with $\mathrm{OAC}$, and most would experience no events ${ }^{12}$. $\mathrm{CHA}_{2} \mathrm{DS}_{2}$-VASc also does not incorporate all possible risk factors for embolism, such as renal impairment, nor detailed echocardiographic or biochemical parameters ${ }^{13}$.

On the other hand, OAC increases the risk of bleeding complications, the most serious of which is intracranial haemorrhage ${ }^{4}$. Many stroke risk factors have also been identified as risk factors for bleeding ${ }^{10,14}$. Several risk stratification scores have been developed to estimate bleeding risk in $\mathrm{AF}^{15}$. For example, the HAS-BLED score (Hypertension, Abnormal renal/liver function, previous Stroke, previous Bleeding/predisposition, Labile INR, Elderly (age $\geq 65$ ), concomitant Drugs or alcohol abuse), to assess the risk of major bleeding, and draw attention to revisable bleeding risk factors ${ }^{16}$.

Continuous efforts have been made to improve stroke and bleeding risk stratification in AF and various studies point out to a promising role of cardiac biomarkers to further refine these risks ${ }^{17,18}$.

Plasma glycoprotein von Willebrand factor (vWF) is synthesized mainly by endothelial cells in response to endothelial activation or damage, promoting platelet adhesion and aggregation at sites of vascular injury ${ }^{19}$. vWF has been considered an established marker of endothelial damage/dysfunction ${ }^{20}$. Increased plasma levels of vWF have been found in different inflammatory and atherosclerotic vascular diseases ${ }^{21}$, as well as in $\mathrm{AF}^{22,23}$, and is predictive of stroke and vascular events ${ }^{24}$. We have previously shown that high plasma vWF predicts adverse cardiovascular events, mortality and major bleeding in anticoagulated AF patients ${ }^{25}$.

In this study, we investigated the role of vWF on prognosis in relation to cardiovascular events, stroke and cardiovascular mortality, as well as major bleeding, in a large prospective 'real-world' cohort of anticoagulated patients with AF, and determined whether the addition of vWF to current clinical risk stratification schemes improved event-risk prediction.

\section{Methods}

Study patients. During the second semester of 2007 we recruited consecutive patients with non-valvular AF from our outpatient anticoagulation clinic in a tertiary hospital of south-eastern Spain. All the patients received VKA and needed to be stabilized for at least 6 months (international normalized ratio: 2.0-3.0), so at baseline the average time in therapeutic range (TTR) was $100 \%$, to enable 'anticoagulation status' homogeneity of the baseline cohort. We excluded patients with valvular AF or prosthetic heart valves, as well as those with any acute coronary syndrome, stroke, hemodynamic instability, hospital admissions or surgical interventions in the preceding 6 months.

Data on baseline clinical characteristics were recorded at study entry. The $\mathrm{CHA}_{2} \mathrm{DS}_{2}$-VASc and the HAS-BLED scores were calculated using established definitions of the different risk factors, as previously described ${ }^{8,26}$. Follow-up was conducted by visits to our anticoagulation clinic. Adverse cardiovascular events were defined as follows: stroke/transient ischaemic attack, systemic and peripheral embolism, acute coronary syndrome, acute heart failure and cardiac death. The composite cardiovascular endpoint included all these events. Major bleeding events were assessed following the International Society of Thrombosis and Haemostasis criteria ${ }^{27}$. All-cause deaths were also recorded.

Blood samples and laboratory analysis. At study inclusion, blood samples were drawn atraumatically and without stasis into syringes pre-loaded with trisodium citrate $(0.011 \mathrm{~mol} / \mathrm{l})$. Platelet-poor plasma fractions were obtained by centrifugation at $4^{\circ} \mathrm{C}$ for $20 \mathrm{~min}$ at $2,200 \mathrm{~g}$. Aliquots were stored at $-80^{\circ} \mathrm{C}$ to allow batch analysis. Plasma vWF antigen levels were assessed in an automated coagulometer ACL Top 3G, HemosIL von Willebrand factor (Instrumentation Laboratory, Lexington, Massachusetts). The inter-assay and intra-assay coefficients of variation were $1.4 \%$ and $9 \%$, respectively, and the lower limits of detection were $2.2 \mathrm{IU} / \mathrm{dl}$ and $21 \mathrm{ng} / \mathrm{ml}$, respectively.

Statistical analysis. Continuous variables were tested for normal distribution by the Kolmogorov-Smirnov test. Continuous variables are presented as a mean \pm standard deviation or median (interquartile range), as appropriate, and categorical variables, as percentages. Receiver-operator characteristic (ROC) curve analyses (ie. c-indexes) were generated to test the predictive discrimination of vWF to identify association with adverse events during follow-up. The cut point with the best sensitivity and specificity for each adverse event was chosen. The independent effect of variables (vWF, $\mathrm{CHA}_{2} \mathrm{DS}_{2}$-VASc and HAS-BLED scores) on prognosis was calculated using a Cox proportional hazards regression model. Differences in event-free survival between patients with different levels of vWF were reflected by Kaplan-Meier curves.

To compare the predictive ability of risk-stratification schemes before and after adding vWF levels to the models, we calculated the statistical significance of the difference between the areas under the two ROC curves (AUC) with the method of DeLong et al. ${ }^{28}$. Also, improvement in the predictive accuracy of the models was evaluated by calculating the relative integrated discrimination improvement (IDI) and the net reclassification improvement (NRI), as described by Pencina et al. ${ }^{29}$.

We also estimated the clinical usefulness and net benefit of the new predictive models using decision curve analysis (DCA), as described by Vickers et al. ${ }^{30}$. This analysis identifies patients who will have any of the adverse events evaluated, based on the predictions of the modified risk score in comparison with the original. The $\mathrm{x}$-axis shows threshold values for adverse event risk while the y-axis represents the net benefit for the different threshold values of adverse event risk. A higher net benefit is provided by those prediction models that are farthest away from the slanted dash grey line (i.e., assume all adverse events) and the horizontal black line (i.e., assume no adverse event).

A p value $<0.05$ was accepted as statistically significant. Statistical analyses were performed using SPSS v. 15.0 (SPSS, Inc., Chicago, IL, USA), MedCalc v. 16.4.3 (MedCalc Software bvba, Ostend, Belgium) and STATA v. 12.0 (Stata Corp., College Station, TX, USA) for Windows. 


\begin{tabular}{|l|c|}
\hline & Median (IQR) N (\%) \\
\hline Age & $76(71-81)$ \\
\hline Male sex & $599(49.3)$ \\
\hline Hypertension & $1002(82.5)$ \\
\hline Diabetes & $320(26.4)$ \\
\hline Heart failure & $378(31.1)$ \\
\hline Ischaemic heart disease & $231(19.0)$ \\
\hline Smoking & $183(15.1)$ \\
\hline Dyslipemia & $393(32.3)$ \\
\hline Previous stroke & $224(18.4)$ \\
\hline Previous bleeding & $102(8.4)$ \\
\hline Alcohol abuse & $39(3.2)$ \\
\hline Renal disease & $125(10.3)$ \\
\hline Hepatic disease & $14(1.2)$ \\
\hline Antiplatelet drugs & $216(17.8)$ \\
\hline CHA ${ }_{2}$ DS ${ }_{2}$-VASc score & $4(3-5)$ \\
\hline HAS-BLED score & $2(2-3)$ \\
\hline vWF levels (UI/dL) & $189.7(132.0-234.0)$ \\
\hline
\end{tabular}

Table 1. Baseline characteristics of atrial fibrillation patients $(\mathrm{N}=1215)$. IQR: interquartile range.

\begin{tabular}{|l|c|c|}
\hline Composite cardiovascular end-point & n (\%) & Annual rate (\%) \\
\hline Stroke & $226(18.6)$ & 2.90 \\
\hline Total mortality & $115(9.5)$ & 1.45 \\
\hline Cardiovascular death & $498(41.0)$ & 6.30 \\
\hline Major bleeding & $76(6.3)$ & 0.96 \\
\hline Intracranial haemorrhage & $222(18.3)$ & 2.81 \\
\hline Mortality of bleeding episodes & $65(5.4)$ & 0.82 \\
\hline
\end{tabular}

Table 2. Annual rate of the different adverse events. *Stroke-thromboembolism, acute coronary syndrome, acute heart failure and cardiovascular death.

Ethical issues. This study was approved by the Ethical Committee of Morales Meseguer University Hospital and was performed in accordance with the ethical standards laid down in the 1964 Declaration of Helsinki and its later amendments. Patients gave their informed consent prior to their inclusion in the study.

\section{Results}

We included 1215 patients with non-valvular AF. Baseline characteristics of the patients are shown in Table 1. During a median follow-up of 2373 (IQR 1564-2892) days, 115 patients presented stroke (1.45\%/year); 498 patients died (6.30\%/year) and 222 experienced major bleeding (2.81\%/year). Annual rates of the different adverse events are summarized in Table 2.

Univariate and multivariate analysis. For each adverse event, we constructed a ROC curve for vWF levels, that gave a median cut-off point of $190 \mathrm{UI} / \mathrm{dL}$ [AUC: 0.60 (95\%CI: 0.56-0.64); $\mathrm{p}<0.001$ ] for the composite cardiovascular end-point; $194 \mathrm{UI} / \mathrm{dL}$ [AUC: 0.60 (95\%CI: 0.55-0.65); $\mathrm{p}<0.001$ ] for stroke; 184 UI/dL [AUC: 0.62 (95\%CI: 0.59-0.65); $\mathrm{p}<0.001$ ] for total mortality; $184 \mathrm{UI} / \mathrm{dL}$ [AUC: 0.64 (95\%CI: 0.57-0.71); $\mathrm{p}<0.001$ ] for cardiovascular mortality and $197 \mathrm{UI} / \mathrm{dL}$ [AUC: 0.61 (95\%CI: 0.57-0.65); $\mathrm{p}<0.001$ ] for major bleeding.

Univariate and multivariate analysis for the different adverse events for vWF levels and stroke and bleeding risk-stratification scores are shown in Table 3. On univariate analyses, plasma vWF levels were significantly predictive of cardiovascular events, stroke, all cause-mortality, cardiovascular death and major haemorrhage. $\mathrm{CHA}_{2} \mathrm{DS}_{2}$-VASc score significantly predicted the composite cardiovascular end-point, stroke, all-cause and cardiovascular death, whilst HAS-BLED score was an independent predictor of major bleeding. After adjustment for the $\mathrm{CHA}_{2} \mathrm{DS}_{2}$-VAScscore, vWF levels were significantly associated with the incidence of the composite cardiovascular end-point, stroke, all-cause mortality and cardiovascular mortality. Also, when we adjusted for HAS-BLED score, vWF was a significant predictor of major bleeding (Table 3). Figure 1 illustrates Kaplan-Meier plots for each outcome in relation to vWF levels (please see online supplement).

Additive effect of von Willebrand factor levels to clinical risk scores. Based on IDI and NRI, the addition of vWF to $\mathrm{CHA}_{2} \mathrm{DS}_{2}$-VASc statistically improved its predictive value for cardiovascular events, stroke and cardiovascular mortality, but C-indexes were not significantly different and remained modest (approx. 0.6). 


\begin{tabular}{|c|c|c|c|}
\hline & & Univariate analysis $\mathrm{HR}(95 \% \mathrm{CI})$ & Multivariate analysis HR (95\% CI) \\
\hline \multirow{2}{*}{ Composite cardiovascular end-point ${ }^{1}$} & $\mathrm{CHA}_{2} \mathrm{DS}_{2}$-VASc score & $1.36(1.26-1.48) ; \mathrm{p}<0.001$ & $1.34(1.23-1.46) ; \mathrm{p}<0.001$ \\
\hline & $\mathrm{vWF}>190 \mathrm{UI} / \mathrm{dL}$ & $1.96(1.50-2.57) ; \mathrm{p}<0.001$ & $1.77(1.35-2.32) ; \mathrm{p}<0.001$ \\
\hline \multirow{2}{*}{ Stroke $^{1}$} & $\mathrm{CHA}_{2} \mathrm{DS}_{2}$-VASc score & $1.35(1.20-1.51) ; \mathrm{p}<0.001$ & $1.32(1.18-1.49) ; \mathrm{p}<0.001$ \\
\hline & $\mathrm{vWF}>194 \mathrm{UI} / \mathrm{dL}$ & $2.19(1.50-3.20) ; \mathrm{p}<0.001$ & $1.98(1.36-2.90) ; \mathrm{p}<0.001$ \\
\hline \multirow{2}{*}{ All-cause mortality ${ }^{1}$} & $\mathrm{CHA}_{2} \mathrm{DS}_{2}$-VASc score & $1.33(1.26-1.40) ; \mathrm{p}<0.001$ & $1.30(1.23-1.38) ; \mathrm{p}<0.001$ \\
\hline & $\mathrm{vWF}>184 \mathrm{UI} / \mathrm{dL}$ & $1.75(1.46-2.10) ; \mathrm{p}<0.001$ & $1.53(1.28-1.84) ; \mathrm{p}<0.001$ \\
\hline \multirow{2}{*}{ Cardiovascular death $^{1}$} & $\mathrm{CHA}_{2} \mathrm{DS}_{2}$-VASc score & $1.49(1.30-1.71) ; \mathrm{p}<0.001$ & $1.46(1.27-1.68) ; \mathrm{p}<0.001$ \\
\hline & $\mathrm{vWF}>184 \mathrm{UI} / \mathrm{dL}$ & $2.34(1.44-3.80) ; \mathrm{p}<0.001$ & $1.95(1.20-3.18) ; \mathrm{p}=0.007$ \\
\hline \multirow{2}{*}{ Major bleeding ${ }^{2}$} & HAS-BLED score & $1.45(1.28-1.65) ; \mathrm{p}<0.001$ & $1.39(1.23-1.58) ; \mathrm{p}<0.001$ \\
\hline & vWF $>197 \mathrm{UI} / \mathrm{dL}$ & $2.13(1.62-2.79) ; \mathrm{p}<0.001$ & $1.95(1.49-2.57) ; \mathrm{p}<0.001$ \\
\hline
\end{tabular}

Table 3. Cox analysis for vWF levels, the $\mathrm{CHA}_{2} \mathrm{DS}_{2}$-VASc and the HAS-BLED scores for the different endpoints. HR: hazard ratio. ${ }^{1}$ Variables included in multivariate analysis: $\mathrm{CHA}_{2} \mathrm{DS}_{2}$-VASc score and vWF plasma levels. ${ }^{2}$ Variables included in multivariate analysis: HAS-BLED score and vWF plasma levels.

Whilst statistically significant, the change in IDI and NRI was small ( $<1 \%$ increase) in its predictive ability for cardiovascular events, stroke and cardiovascular mortality (Table 4). For major bleeding, addition of vWF to HAS-BLED improved the c-index but not the IDI or NRI (Table 5).

DCA graphically shows minimal net benefit of the modified $\mathrm{CHA}_{2} \mathrm{DS}_{2}$-VASc and HAS-BLED scores, that does not justify their clinical use or impact on practical decision-making (Fig. 1).

\section{Discussion}

In this study of a large contemporary 'real world' cohort of stable anticoagulated non-valvular AF patients, we show that even if vWF acts as a simple prognostic biomarker and that the addition of vWF levels to the $\mathrm{CHA}_{2} \mathrm{DS}_{2}$-VASc and the HAS-BLED scores statistically improved prediction for some endpoints, absolute changes and clinical improvement or impact on clinical decision-making (using DCA) was marginal, with c-indexes all remaining modest (approx. 0.6).

Cardiac biomarkers have evolved as prognostic tools in different cardiovascular scenarios. The importance of vWF to cardiovascular disorders has been long recognised ${ }^{31}$. Raised levels of vWF have been described in AF individuals, compared to those in sinus rhythm ${ }^{23}$. Also, the prognostic role of plasma vWF in AF has been well-established ${ }^{32,33}$. In the Rotterdam Study, which included more than 6000 participants, the risk of stroke was associated with vWF levels in the general population, no matter AF was present or not ${ }^{34}$. Also, vWF appears to be able to identify, not only patients with high thromboembolic risk, but also subjects at risk of major bleeding and mortality. Indeed, major bleedings and death occur more frequently than embolism in anticoagulated $\mathrm{AF}$ patients, and in this study we show that bleeding rates doubled rates of stroke, and mortality was four times greater.

Other biomarkers, such us Growth Differentiation factor-15 (GDF-15, a marker of oxidative stress and inflammation) and high-sensitivity Troponin $\mathrm{T}$ (hs- $\mathrm{TnT}$ ), have also been shown to act as additive prognostic markers (at least statistically) for major bleeding and death in AF subjects with $\mathrm{OAC}^{35,36}$.

In the present study, we demonstrate that raised vWF levels doubled the risk of stroke, cardiovascular death and major bleeding, and increased (by more than 50\%) cardiovascular events and all-cause mortality. We have previously shown that this biomarker acts as an independent risk factor for adverse events, including cardiovascular events (stroke, acute coronary syndrome and acute heart failure), cardiovascular death, all cause mortality and major haemorrhagic episodes in a 'real-life' anticoagulated non-valvular AF population ${ }^{25}$. In another study, raised vWF activation factor levels (a modified test that detects vWF with high platelet affinity) correlated with all-cause mortality and cardiovascular events, but no association was observed with bleeding complications ${ }^{37}$. The same group had previously described an increased risk of bleeding complications, cardiovascular and all-cause mortality in patients with high levels of vWF during treatment with warfarin; nonetheless, only one third of the subjects in both studies had AF as the indication for $\mathrm{OAC}^{38}$.

Currently, the $\mathrm{CHA}_{2} \mathrm{DS}_{2}$-VASc score is recommended to assist clinical decision-making for OAC ${ }^{6,7}$, but its predictive value is modest $^{8}$. In order to improve stroke risk stratification, composite risk prediction scores $\left(\mathrm{CHA}_{2} \mathrm{DS}_{2}-\mathrm{VASc}+\mathrm{HAS}-\mathrm{BLED}\right.$ or $\left.\mathrm{CHADS}_{2}+\mathrm{HAS}-\mathrm{BLED}\right)$ have been compared with separate individual scores, but they have not shown to significantly improve clinical prediction of thromboembolism and bleeding $^{39}$. Unsurprisingly, cardiac biomarkers could help to refine event-risk stratification in these patients. For example, Lip et al. first showed that plasma vWF levels added to the $\mathrm{CHADS}_{2}$ and the Birmingham (precursor of $\mathrm{CHA}_{2} \mathrm{DS}_{2}$-VASc) schemes could help to refine risk stratification for vascular events (ischemic stroke, myocardial infarction or vascular death) in AF patients enrolled in the SPAF III trial ${ }^{17}$; however, they were not able to identify an independent association with the risk of stroke. Recently, Hijazi et al. demonstrated that N-terminal fragment B-type Natriuretic Peptide (NT-proBNP) and hs-TnT improved risk stratification for stroke, cardiac death and bleeding beyond the $\mathrm{CHA}_{2} \mathrm{DS}_{2}$-VASc score ${ }^{36,40}$. This group then proposed the ABC score (which adds biomarkers to clinical factors) in a selected clinical trial cohort of AF patients anticoagulated with apixaban or warfarin from the ARISTOTLE (Apixaban for Reduction in Stroke and Other Thromboembolic Events in Atrial Fibrillation) ${ }^{41}$. In a similar manner, the $\mathrm{ABC}$-bleeding score was also validated, including biomarkers (haemoglobin, hsTnT and GDF-15 or cystatin C/glomerular filtration rate) and this score performed better than the (clinical 

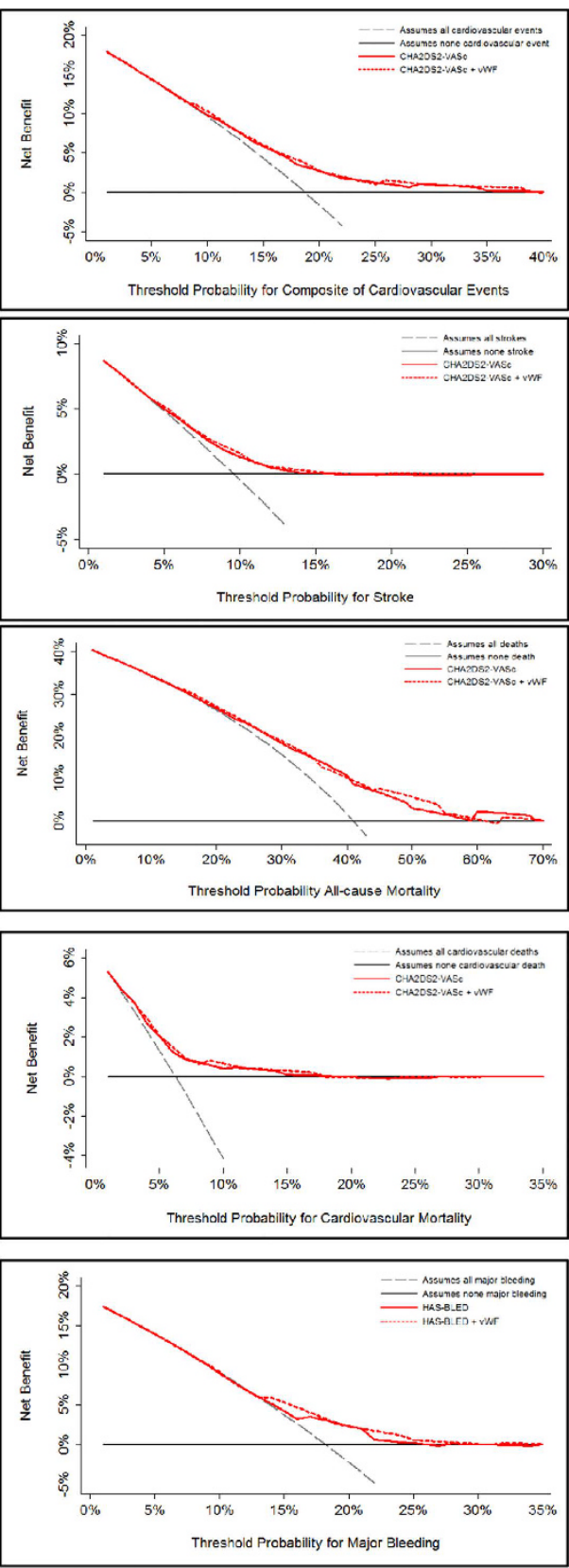

(B) Composite of cardiovascular events.

(A) Stroke.

(C) All-cause mortality.

(D) Cardiovascular mortality.

Figure 1. Decision curves for the original and modified risk scores (adding von Willebrand factor).

factor-based) HAS-BLED or ORBIT scores ${ }^{18}$. Whilst statistically significant improvement in prediction was seen, overall c-indexes still remain modest (approx. 0.6-0.7).

Contrary to this evidence from the highly selected trial patient cohorts, we have not been able to demonstrate that the addition of a biomarker (specifically, vWF) to clinical risk scores markedly improves decision making for event risk prediction, as DCA only showed minimal net benefit of the modifiedscores that incorporated biomarkers. Nonetheless, our study adds some novel strengths to the current knowledge in this field, amongst 'real-world' AF patients. In light of our data, even if vWF levels provide independent prognostic information, we cannot recommend the addition of $\mathrm{vWF}$ as a biomarker to the $\mathrm{CHA}_{2} \mathrm{DS}_{2}$-VASc and HAS-BLED risk scores in order to refine event-risk prediction.

More insights are needed to explore the role of cardiac biomarkers to aid clinical decision-making for thromboprophylaxis in AF. The continued preoccupation with trying to improve prediction of 'high risk' patients with ever more complex scores (and often multiple biomarkers), with only marginal improvement in predictive performance, at the cost of simplicity and practicability, would seem counterintuitive for everyday clinical management $^{42}$. Meanwhile, a more simple strategy, using conventional clinical risk scores, should be the preferred option. In short, simplicity and practicability (and costs of biomarkers) should be balanced. 


\begin{tabular}{|c|c|c|c|c|c|c|c|}
\hline & C-index & $\begin{array}{c}95 \% \mathrm{CI} \\
p\end{array}$ & $p^{*}$ & IDI & $p$ & NRI & $p$ \\
\hline \multicolumn{8}{|c|}{ (A) Composite cardiovascular end-point } \\
\hline $\mathrm{CHA}_{2} \mathrm{DS}_{2}$-VASc & 0.630 & $\begin{array}{l}0.603-0.658 \\
<0.001\end{array}$ & \multirow{2}{*}{0.111} & \multirow{2}{*}{0.0065} & \multirow{2}{*}{$<0.001$} & \multirow{2}{*}{-0.016} & \multirow{2}{*}{0.012} \\
\hline $\mathrm{CHA}_{2} \mathrm{DS}_{2}-\mathrm{VASc}+\mathrm{vWF}$ & 0.641 & $\begin{array}{c}0.613-0.668 \\
<0.001\end{array}$ & & & & & \\
\hline \multicolumn{8}{|l|}{ (B) Stroke } \\
\hline $\mathrm{CHA}_{2} \mathrm{DS}_{2}-\mathrm{VASc}$ & 0.610 & $\begin{array}{c}0.582-0.637 \\
\mathrm{p}<0.001\end{array}$ & \multirow{2}{*}{0.131} & \multirow{2}{*}{0.0037} & \multirow{2}{*}{0.040} & \multirow{2}{*}{-0.017} & \multirow{2}{*}{$<0.001$} \\
\hline $\mathrm{CHA}_{2} \mathrm{DS}_{2}-\mathrm{VASc}+\mathrm{vWF}$ & 0.623 & $\begin{array}{c}0.595-0.650 \\
\mathrm{p}<0.001\end{array}$ & & & & & \\
\hline \multicolumn{8}{|l|}{ (C) All-cause mortality } \\
\hline $\mathrm{CHA}_{2} \mathrm{DS}_{2}-\mathrm{VASc}$ & 0.661 & $\begin{array}{c}0.634-0.688 \\
\mathrm{p}<0.001\end{array}$ & \multirow{2}{*}{0.076} & \multirow{2}{*}{0.0091} & \multirow{2}{*}{0.048} & \multirow{2}{*}{0.001} & \multirow{2}{*}{0.864} \\
\hline $\mathrm{CHA}_{2} \mathrm{DS}_{2}-\mathrm{VASc}+\mathrm{vWF}$ & 0.670 & $\begin{array}{c}0.643-0.697 \\
\mathrm{p}<0.001\end{array}$ & & & & & \\
\hline \multicolumn{8}{|l|}{ (D) Cardiovascular mortality } \\
\hline $\mathrm{CHA}_{2} \mathrm{DS}_{2}$-VASc & 0.656 & $\begin{array}{c}0.628-0.683 \\
\mathrm{p}<0.001\end{array}$ & \multirow{2}{*}{0.156} & \multirow{2}{*}{0.0041} & \multirow{2}{*}{0.033} & \multirow{2}{*}{-0.018} & \multirow{2}{*}{0.004} \\
\hline $\mathrm{CHA}_{2} \mathrm{DS}_{2}-\mathrm{VASc}+\mathrm{vWF}$ & 0.670 & $\begin{array}{c}0.643-0.697 \\
p<0.001\end{array}$ & & & & & \\
\hline
\end{tabular}

Table 4. Comparison of the ROC curve, IDI and NRI of the modified $\mathrm{CHA}_{2} \mathrm{DS}_{2}-\mathrm{VASc} v$ s. original $\mathrm{CHA}_{2} \mathrm{DS}_{2}$-VASc in predicting endpoints. $\mathrm{CI}=$ confidence interval; IDI = integrated discriminatory improvement; NRI $=$ net reclassification improvement. ${ }^{*}$ For c-index comparison.

\begin{tabular}{|l|c|c|c|c|c|c|c|}
\hline & C-index & $\begin{array}{c}\mathbf{9 5 \%} \text { CI } \\
\boldsymbol{p}\end{array}$ & $\boldsymbol{p}^{*}$ & IDI & $\boldsymbol{p}$ & NRI & $\boldsymbol{p}$ \\
\hline HAS-BLED & 0.592 & $\begin{array}{c}0.564-0.620 \\
\mathrm{p}<0.001\end{array}$ & \multirow{2}{*}{0.025} & 0.0105 & 0.056 & 0.012 & 0.735 \\
\cline { 1 - 4 } HAS-BLED+vWF & 0.614 & $\begin{array}{c}0.586-0.641 \\
\mathrm{p}<0.001\end{array}$ & & & \\
\hline
\end{tabular}

Table 5. Comparison of the ROC curve, IDI and NRI of the modified HAS-BLED vs. original HAS-BLED in predicting major bleeding. $\mathrm{CI}=$ confidence interval; $\mathrm{IDI}=$ integrated discriminatory improvement; $\mathrm{NRI}=$ net reclassification improvement. ${ }^{*}$ For c-index comparison.

\section{Limitations}

Only a single determination of plasma vWF levels was made, at study entry, thus we cannot be sure if making other determinations during follow-up could have changed our results. Only patients on VKA were included, so our results may not be generalized to individuals receiving NOACs. TTR was $100 \%$ at study entry, but we do not have data of this parameter over the follow-up period, which could affect the occurrence of the adverse events. Although vWF levels are known to be associated to cardiovascular risk factors (such as hypertension or heart failure), we have previously shown that the association of this biomarker with adverse events in AF is independent of other clinical variables ${ }^{25}$.

\section{Conclusions}

In conclusion, $\mathrm{vWF}$ acts as a simple prognostic biomarker in anticoagulated AF patients and, whilst addition of vWF levels to the $\mathrm{CHA}_{2} \mathrm{DS}_{2}$-VASc and the HAS-BLED scores statistically improved prediction for some endpoints, the absolute changes and clinical value or impact on practical decision-making was minimal.

\section{References}

1. Wolf, P. A., Dawber, T. R., Thomas, H. E. \& Kannel, W. B. Epidemiologic assessment of chronic atrial fibrillation and risk of stroke: the Framingham study. Neurology. 28, 973-977 (1978).

2. Benjamin, E. J. et al. Impact of atrial fibrillation on the risk of death: the Framingham Heart Study. Circulation. 98, 946-952 (1998).

3. Senoo, K., Lip, G. Y., Lane, D. A., Büller, H. R. \& Kotecha, D. Residual risk of stroke and death in anticoagulated patients according to the type of atrial fibrillation: AMADEUS Trial. Stroke. 46, 2523-2528 (2015).

4. Hart, R. G., Pearce, L. A. \& Aguilar, M. I. Meta-analysis: antithrombotic therapy to prevent stroke in patients who have nonvalvular atrial fibrillation. Ann. Intern. Med. 146, 857-867 (2007).

5. Ruff, C. T. et al. Comparison of the efficacy and safety of new oral anticoagulants with warfarin in patients with atrial fibrillation: a meta-analysis of randomised trials. Lancet. 383, 955-962 (2014).

6. Camm, A. J. et al. 2012 focused update of the ESC Guidelines for the management of atrial fibrillation: an update of the 2010 ESC Guidelines for the management of atrial fibrillation-developed with the special contribution of the European Heart Rhythm Association. Europace. 14, 1385-1413 (2012).

7. January, C. T. et al. AHA/ACC/HRS guideline for the management of patients with atrial fibrillation: a report of the American College of Cardiology/American Heart Association Task Force on Practice Guidelines and the Heart Rhythm Society. J. Am. Coll. Cardiol. 64, e1-76 (2014). 
8. Lip, G. Y., Nieuwlaat, R., Pisters, R., Lane, D. A. \& Crijns, H. J. Refining clinical risk stratification for predicting stroke and thromboembolism in atrial fibrillation using a novel risk factor-based approach: the euro heart survey on atrial fibrillation. Chest. 137, 263-272 (2010).

9. Jover, E. et al. Predictive value of the CHA2DS2-VASc score in atrial fibrillation patients at high risk for stroke despite oral anticoagulation. Rev. Esp. Cardiol. 65, 627-633 (2012).

10. Lip, G. Y. Stroke and bleeding risk assessment in atrial fibrillation: when, how, and why? Eur. Heart J. 34, 1041-1049 (2013).

11. Coppens, M. et al. The CHA2DS2-VASc score identifies those patients with atrial fibrillation and a CHADS2 score of 1 who are unlikely to benefit from oral anticoagulant therapy. Eur. Heart J. 34, 170-176 (2013).

12. Olesen, J. B. et al. Validation of risk stratification schemes for predicting stroke and thromboembolism in patients with atrial fibrillation: nationwide cohort study. BMJ. 342, d124 (2011).

13. Szymanski, F. M. et al. Stroke risk factors beyond the $\mathrm{CHA}_{2} \mathrm{DS}_{2}$-VASc score: Can we improve our identification of "high stroke risk" patients with atrial fibrillation? Am. J. Cardiol. 116, 1781-1788 (2015).

14. Hughes, M. \& Lip, G. Y. Risk factors for anticoagulation-related bleeding complications in patients with atrial fibrillation: a systematic review. QJM. 100, 599-607 (2007).

15. Lip, G. Y. et al. Bleeding risk assessment and management in atrial fibrillation patients: a position document from the European Heart Rhythm Association, endorsed by the European Society of Cardiology Working Group on Thrombosis. Europace. 13, 723-46 (2011)

16. Lip, G. Y. \& Lane, D. A. Assessing bleeding risk in atrial fibrillation with the HAS-BLED and ORBIT scores: clinical application requires focus on the reversible bleeding risk factors. Eur. Heart J. 36, 3265-3267 (2015).

17. Lip, G. Y., Lane, D., Van Walraven, C. \& Hart, R. G. Additive role of plasma von Willebrand factor levels to clinical factors for risk stratification of patients with atrial fibrillation. Stroke. 37, 2294-2300 (2006).

18. Hijazi, Z. et al. The novel biomarker-based ABC (age, biomarkers, clinical history)-bleeding risk score for patients with atrial fibrillation: a derivation and validation study. Lancet. 387, 2302-11 (2016).

19. Sadler, J. E. Biochemistry and genetics of von Willebrand factor. Annu. Rev. Biochem. 67, 395-424 (1998).

20. Blann, A. von Willebrand factor and the endothelium in vascular disease. Br J Biomed Sci. 50, 125-134 (1993).

21. Blann, A. D. \& Lip, G. Y. The endothelium in atherothrombotic disease: assessment of function, mechanisms and clinical implications. Blood Coagul. Fibrinolysis. 9, 297-306 (1998).

22. Marín, F. et al. Plasma von Willebrand factor, soluble thrombomodulin, and fibrin D-dimer concentrations in acute onset nonrheumatic atrial fibrillation. Heart. 90, 1162-1166 (2004).

23. Lip, G. Y., Lowe, G. D., Rumley, A. \& Dunn, F. G. Increased markers of thrombogenesis in chronic atrial fibrillation: effects of warfarin treatment. Br. Heart J. 73, 527-533 (1995).

24. Conway, D. S., Pearce, L. A., Chin, B. S., Hart, R. G. \& Lip G. Y. Prognostic value of plasma von Willebrand factor and soluble P-selectin as indices of endothelial damage and platelet activation in 994 patients with nonvalvular atrial fibrillation. Circulation. 107, 3141-3145 (2003).

25. Roldán, V. et al. Plasma von Willebrand factor levels are an independent risk factor for adverse events including mortality and major bleeding in anticoagulated atrial fibrillation patients. J. Am. Coll. Cardiol. 57, 2496-5204 (2011).

26. Pisters, R. et al. A novel user-friendly score (HAS-BLED) to assess 1-year risk of major bleeding in patients with atrial fibrillation: the Euro Heart Survey. Chest. 138, 1093-100 (2010).

27. Schulman, S. \& Kearon C. Definition of major bleeding in clinical investigations of antihemostatic medicinal products in nonsurgical patients. J. Thromb. Haemost. 3, 692-694 (2005).

28. DeLong, E. R., DeLong, D. M. \& Clarke-Pearson, D. L. Comparing the areas under two or more correlated receiver operating characteristic curves: a nonparametric approach. Biometrics. 44, 837-45 (1988).

29. Pencina, M. J., D’Agostino, R. B. \& Vasan, R. S. Evaluating the added predictive ability of a new marker: from area under the ROC curve to reclassification and beyond. Stat. Med. 27, 157-172 (2008).

30. Vickers, A. J. \& Elkin, E. B. Decision curve analysis: a novel method for evaluating prediction models. Med. Decis. Making. 26, 565-574 (2006)

31. Lip, G. Y. \& Blann, A. D. von Willebrand factor and its relevance to cardiovascular disorders. Br. Heart J. 74, 580-3 (1995).

32. Freynhofer, M. K. et al. Prognostic value of plasma von Willebrand factor and its cleaving protease ADAMTS13 in patients with atrial fibrillation. Int. J. Cardiol. 168, 317-25 (2013).

33. Krishnamoorthy, S. et al. Prognostic role of plasma von Willebrand factor and soluble E-selectin levels for future cardiovascular events in a 'real-world' community cohort of patients with atrial fibrillation. Eur. J. Clin. Invest. 43, 1032-8 (2013).

34. Wieberdink, R. G. et al. High von Willebrand factor levels increase the risk of stroke: the Rotterdam study. Stroke. 41, 2151-6 (2010).

35. Wallentin, L. et al. Growth differentiation factor 15, a marker of oxidative stress and inflammation, for risk assessment in patients with atrial fibrillation: insights from the apixaban for reduction in stroke and other thromboembolic events in atrial fibrillation (ARISTOTLE) trial. Circulation. 130, 1847-1858 (2014).

36. Hijazi, Z. et al. High-sensitivity troponin T and risk stratification in patients with atrial fibrillation during treatment with apixaban or warfarin. J. Am. Coll. Cardiol. 63, 52-61 (2014).

37. Lind, M., Johansson, L., Nilsson, T. K., Jansson, J. H. \& Hollestelle, M. J. von Willebrand activation factor as a marker of mortality, cardiovascular events, and bleeding complications in patients treated with oral anticoagulants. Thromb Res. 136, 878-82 (2015).

38. Lind, M. et al. Von Willebrand factor predicts major bleeding and mortality during oral anticoagulant treatment. J. Intern. Med. 271, 239-46 (2012)

39. Banerjee, A., Fauchier, L., Bernard-Brunet, A., Clementy, N. \& Lip G. Y. Composite risk scores and composite endpoints in the risk prediction of outcomes in anticoagulated patients with atrial fibrillation. The Loire Valley atrial fibrillation project. Thromb Haemost. 111, 549-556 (2014).

40. Hijazi, Z. et al. N-terminal pro-B-type natriuretic peptide for risk assessment in patients with atrial fibrillation: insights from the ARISTOTLE Trial (Apixaban for the prevention of stroke in subjects with atrial fibrillation). J. Am. Coll. Cardiol. 61, 2274-2284 (2013).

41. Hijazi, Z. et al. The ABC (age, biomarkers, clinical history) stroke risk score: a biomarker-based risk score for predicting stroke in atrial fibrillation. Eur. Heart J. 37, 1582-1590 (2016).

42. Lip, G. Y. \& Lane, D. A. Bleeding risk assessment in atrial fibrillation: observations on the use and misuse of bleeding risk scores. J. Thromb. Haemost, doi: 10.1111/jth.13386 (2016).

\section{Acknowledgements}

This work was supported by ISCIII and FEDER (PI13/00513 and P14/00253), Fundación Séneca (grant number: 19245/PI/14), and RD12/0042/0050.

\section{Author Contributions}

A.G.F. wrote the main manuscript text and Tables, V.R. and F.M. are responsible of study design, data acquisition, analysis and interpretation of data, and critical revision of the manuscript, J.M.R.C. prepared Figures and helped with statistical analysis and data interpretation, D.H.R. helped with statistical analysis and data interpretation, M.V. and V.V. revised the manuscript and G.Y.H.L. made a critical revision of the manuscript. 


\section{Additional Information}

Supplementary information accompanies this paper at http://www.nature.com/srep

Competing financial interests: The authors declare no competing financial interests.

How to cite this article: García-Fernández, A. et al. Does von Willebrand factor improve the predictive ability of current risk stratification scores in patients with atrial fibrillation? Sci. Rep. 7, 41565; doi: 10.1038/srep41565 (2017).

Publisher's note: Springer Nature remains neutral with regard to jurisdictional claims in published maps and institutional affiliations.

(c) (i) This work is licensed under a Creative Commons Attribution 4.0 International License. The images or other third party material in this article are included in the article's Creative Commons license, unless indicated otherwise in the credit line; if the material is not included under the Creative Commons license, users will need to obtain permission from the license holder to reproduce the material. To view a copy of this license, visit http://creativecommons.org/licenses/by/4.0/

(C) The Author(s) 2017 